\title{
Unité dans la diversité
}

Il n'y a qu'une vérité, de toute éternité, mais elle apparaît différemment à chacun.

Le fait qu'une vérité existe rend la diversité véridique.

Friedrich Schiller

Schiller disait-il vrai au sujet de la vérité? Cette question peut donner lieu à des débats passionnés, à l'image de ceux en cours dans le BMS et ailleurs sur la «caisse unique». On peut être unanime sur un point: la «vérité» est semblable à une montagne dont la face nord est composée de parois rocheuses abruptes couvertes de glace et la face sud, d'une pente douce recouverte de végétation tropicale. Ou pour les personnes familières de la Suisse orientale et en caricaturant un peu les choses: aux «Churfirsten», ces montagnes dentelées qui surplombent le lac de Walenstadt.

Quelques exemples tirés des lettres de lecteurs publiées dans les éditions précédentes du BMS illustrent cette affirmation: «Une méga-caisse unique aurait tout pouvoir pour nous dicter toutes les conditions. Il ne fait aucun doute qu'elle abuserait de ce pouvoir.» Un collègue estime au contraire que le système actuel génère des exagérations dictatoriales: «La conséquence de la dictature actuelle, c'est, pour nous, la perte de liberté et pour nos patients, l'augmentation de la prime d'assurance et toujours moins de soins de bonne qualité». Sa conclusion logique: «Vive la caisse unique.»

Le fait que cette initiative soulève des questions fondamentales est souligné par les deux parties: «Le vote du 11 mars 2007 est un vote crucial: celui d'un choix de société: sociale ou économique. Quel avenir voulons-nous pour nos enfants, celui d'une société égoïste et soumise aux lois de l'économie privée, ou celui d'une société solidaire et responsable, offrant une garantie de soins à tous?» Et la position contraire: «le système souhaité ne serait non seulement pas plus juste sur le plan médical; il amoindrirait aussi massivement toute incitation à économiser et créerait de nouveaux problèmes, avant tout pour les patients. La caisse unique renforcerait la médecine à deux vitesses en train de se mettre en place. Il en résulterait des injustices inacceptables.»
Thèses et antithèses: on pourrait allonger la liste à volonté. Malgré ce constat, certaines lettres ont reproché à la FMH, donc à l'organisation faittière du corps médical suisse, de s'être «défilée» lorsque la Chambre médicale s'est prononcée pour «la liberté de vote», leurs auteurs considérant cette décision comme un signe de faiblesse et une hésitation à prendre clairement position.

Dans ce contexte, il est intéressant de se demander si la liberté de vote est nécessairement une expression de faiblesse, un signe d'indifférence en faveur du «laissez-faire». L'argumentaire publié par la FMH dans le BMS $n^{\circ} 1 / 2007$ sur la caisse unique, et dans lequel elle présente les différents points de vue de manière approfondie, fait plutôt penser le contraire. A sa lecture, on constate que la liberté de vote peut très bien être vue comme le résultat logique d'un inventaire systématique de la situation actuelle et des solutions de rechange proposées. Le mot d'ordre de la FMH est à comprendre comme un signal très fort. Dans les milieux non médicaux, c'est ce que j'ai du moins ressenti en Suisse alémanique, on s'attendait un peu partout à ce que le corps médical prône le «non» à l'initiative. Comme cela n'a pas été le cas, on s'est interrogé sur la position de la FMH et l'on s'est mis à réfléchir en creusant le sujet.

Si l'on évalue la Chambre médicale à l'aulne des lettres de lecteurs, on constate qu'elle s'est avérée un organe représentatif de l'ensemble du corps médical: elle a décidé la liberté de vote après des débats nourris qui ont reflété un éventail d'opinions aussi étendu que possible. Ce que nous constatons quotidiennement au BMS est apparu au grand jour: on ne peut pas comparer le corps médical, et partant la FMH, à un parti politique dont les membres sont tenus d'exprimer une pensée uniforme sur bien des points. La FMH repose, certes, sur un dénominateur commun constitué par la profession médicale et les obligations qui en résultent face aux patients. Mais, par ailleurs, elle doit également savoir intégrer des intérêts, des positions et des exigences extrêmement divers: une tâche aussi exigeante que passionnante.

Bruno Kesseli 